\title{
EFEITO DO EXTRATO AQUOSO DE Orbignya phalerata (BABAÇU) NA CICATRIZAÇÃO DO ESTÔMAGO EM RATOS: ESTUDO MORFOLÓGICO E TENSIOMÉTRICO
}

\author{
Effect of watery extract of Orbignya phalerata (babassu) in the gastric healing in rats: \\ morfologic and tensiometric study
} \author{
Ferreira $^{4}$ e Alexandre Coutinho Teixeira de Freitas ${ }^{4}$ \\ 1. Trabalho realizado no laboratório de pesquisas do centro de Ciências Biológicas e da Saúde da Universidade Federal do Maranhão \\ 2. Professor do Departamento de Cirurgia da Universidade Federal do Maranhão \\ 3. Médico do Hospital da Universidade Federal do Maranhão \\ 4. Professor Doutor em Cirurgia \\ 5. Aluno de Pós-Graduação - Mestrado \\ 6. Aluno de Pós-Graduação - Doutorado
}

Clelma Pires Batista ${ }^{3}$, Orlando Jorge Martins Torres ${ }^{2}$, Jorge Eduardo F. Matias ${ }^{4}$, Ana Tereza Ramos Moreira ${ }^{4}$, Daniel Colman ${ }^{6}$, João Henrique Felício de Lima ${ }^{6}$, Matheus Martin Macri ${ }^{5}$, Rêmulo José Rauen Jr. ${ }^{5}$, Lydia Masako

\begin{abstract}
RESUMO
Introdução: A utilização de plantas na prevenção e no tratamento de doenças é prática milenar. O babaçu (Orbignya phalerata) é uma palmeira nativa do meio norte do Brasil, tendo sua maior concentração no Estdo do Maranhão. O pó do mesocarpo do coco babaçu é popularmente conhecido como amido e tem sido usado como alimento e como medicamento por apresentar atividade antiinflamatória, imunomoduladora, analgésica e antipirética. Objetivo - Avaliar o efeito do extrato aquoso do mesocarpo de Orbignya phalerata na cicatrização do estômago em ratos, sob aspectos morfológico e tensiométrico. Métodos: Quarenta ratos da linhagem Wistar, adultos, machos foram submetidos à incisão longitudinal de $1 \mathrm{~cm}$ no corpo gástrico e síntese em plano único com pontos separados de polipropilene 6-0. Após este procedimento comum, os animais foram distribuídos aleatoriamente em dois grupos, Orbignya (GO) e Controle (GC), contendo 20 animais cada. No GO utilizou-se dose única intra-peritoneal de extrato aquoso da planta na dose de $50 \mathrm{mg} / \mathrm{kg}$ e no GC, água destilada, $1 \mathrm{ml} / \mathrm{kg}$ de peso. Cada grupo foi dividido em dois subgrupos de 10 , conforme o dia da morte dos animais, nos $3^{\circ}$ e $7^{\circ}$ dias do período pós-operatório. Após a morte, foi realizado o inventário da cavidade abdominal e procedeu-se a retirada do estômago, com posterior avaliação tensiométrica e análise miccccroscópica. Na análise comparativa entre os dois grupos utilizou parâmetros macroscópicos e microscópicos da cicatrização. Resultados: Não foram detectados abscessos, fístulas ou hematomas em nenhum animal. Houve aderências abdominais nos animais dos $3^{\circ}$ e $7^{\circ}$ dias do período pós-operatório nos dois grupos. Houve deiscência da gastrorrafia em um rato do sub-grupo GO morto no $3^{\circ}$ dia. A resistência à insuflação de ar atmosférico foi maior no $\mathrm{GC}$ de três dias $(\mathrm{p}=0,087)$. A análise dos parâmetros histológicos demonstrou diferença estatisticamente significativa apenas quanto à coaptação das bordas, favorecendo o sub-grupo GO de sete dias (p-0,000). Conclusão: O extrato aquoso do mesocarpo de Orbignya phalerata, na dose e via de acesso utilizadas, favoreceu a coaptação das bordas da gastrorrafia nos animais mortos no $7^{\circ}$ dia do período pós-operatório.
\end{abstract}

Descritores: Orbignya phalerata. Cicatrização de Feridas. Estômago. Ratos.

\begin{abstract}
Introduction: The large use of plants in the prevention and treatment of different diseases is a milenar practice. In Brazil due to it's rich flora there is a huge material for research and use in popular medicine. The babassu (Orbignya phalerata) is a native tree from North of the Brazil and has a high concentration at the State of Maranhão. The powder of it's mesocarp is known as amid, and has been used as meal and medication due to the known activity against antiflammatory reaction, pain, pyrexia and immunomodulation. Purpose: To analyze the effect of mesocarp of Orbignya phalerata (babassu) in gastric wound healing in rats under morphologic and tensiometric aspects. Method: Forty Wistar rats, male, adults were submitted to a longitudinal incision of $1 \mathrm{~cm}$ in the gastric body and sutured with separated stitches of polypropilene 6.0. After this common procedure the animals were allocated in the aleatory way in two groups Orbignya (GO) and Control (GC) with 20 animals each one. In the GO was used intraperitoneal doses of 50mg/kh of Orbignya phalerata extract and in the $\mathrm{GC}$ water $1 \mathrm{ml} / \mathrm{Kg}$. Each group was divided in two subgroups of ten rats according to the period of death, at $3 \mathrm{rd}$ and $7 \mathrm{th}$ post-operative days. After the death, the abdominal cavity was evaluated and the stomach withdrawn. For the comparative analysis between the groups the macroscopic and histological parameters of wound healing were utilized. Results: There were no abscess, fistulae and haematom in the animals of the study. Abdominal adhesions were observed in both groups
\end{abstract}


of the animals at 3rd and 7th post-operative days. Dehiscence of the gastrorraphy was identified in one 3rd day rat of subgroups GO. The resistance to air insufflation was higher in GO 3rd day ( $\mathrm{p}=0,087)$. The analysis of histological parameters showed statistical difference related to wound coaptation in favor to subgroup GO 7th post-operative day. Conclusions: The extract of mesocarp of Orbignya phalerata in dose and path utilized positively interfered on the gastrorraphy when compared with control group in animals at 7th post-operative day.

Key Words: Orbignya phalerata. Wound Healing. Stomach. Rats.

\section{Introdução}

A cicatrização é um fenômeno físico, químico e biológico, que ocorre logo após a lesão tecidual, permitindo o restabelecimento da integridade e da função dos tecidos afetados. A descoberta de substâncias que possam modular a resposta à agressão tecidual tem sido objeto de pesquisas. A utilização de plantas na prevenção e no tratamento de doenças é prática milenar. A Orbignya phalerata (babaçu) é uma palmeira encontrada com maior freqüência nas regiões Norte, Nordeste e Centro-Oeste do Brasil, sendo que 54,2\% das palmeiras situam-se no Estado do Maranhão ${ }^{1,2,3,4,5,6}$. Constitui-se de quatro partes: epicarpo, mesocarpo, endocarpo e amêndoas ou sementes ${ }^{7}$. O pó da Orbignya phalerata é obtido a partir do mesocarpo ${ }^{5,6,7,8}$. Apresenta algumas propriedades antiinflamatórias e analgésicas já comprovadas, e tem seu uso popular indicado nos tratamentos de dores menstruais, úlceras, tumores, reumatismo, leucemia, constipação intestinal, colite e obesidade $\mathrm{e}^{6,7,9,10,11,12}$.

Este estudo tem como objetivo avaliar os efeitos do extrato aquoso do mesocarpo de Orbignya phalerata na cicatrização do estômago de ratos, através de uma análise morfológica e tensiométrica.

\section{Método}

Foram utilizados 40 ratos (Rattus novergicus albinus, Rodentia mammalia), da linhagem Wistar, machos, pesando entre 110 e 180 gramas, que. foram ambientados e aclimatados por sete dias para adaptação, recebendo ração padrão para ratos (Purina ${ }^{\circledR}$ Labina,) e água ad libitum .

O mesocarpo de Orbignya phalerata, obtido a partir do coco maduro, depois de seco, foi colocado em estufa à temperatura de 45 a $50^{\circ} \mathrm{C}$, por 24 horas, para retirada da umidade e submetido a processo de moagem obtendo-se o pó. Para obtenção do extrato aquoso, utilizaram-se $10 \mathrm{~g}$ do pó, diluído em 400ml de água destilada, resultando em concentração de $25 \mathrm{mg} / \mathrm{ml}$. Esse extrato final foi acondicionado em frasco de vidro e colocado em refrigerador em temperatura constante de $10^{\circ} \mathrm{C}$ (Figura 1).

Os animais foram submetidos a jejum pré-operatório de 12 horas e pesados. A indução anestésica foi obtida com eter etílico comercial a $97 \%$ em sistema fechado, e mantida em sistema semi-aberto por vaporizador artesanal. Foi realizada uma incisão mediana, longitudinal, medindo $3 \mathrm{~cm}$ de extensão, um centímetro abaixo do processo xifóide, até o peritônio. Foi identificado e exteriorizado o estômago. Em seguida fez-se uma incisão longitudinal de $1 \mathrm{~cm}$ de extensão, na parede anterior do corpo gástrico, próximo à grande curvatura e, por meio de quatro pontos separados,

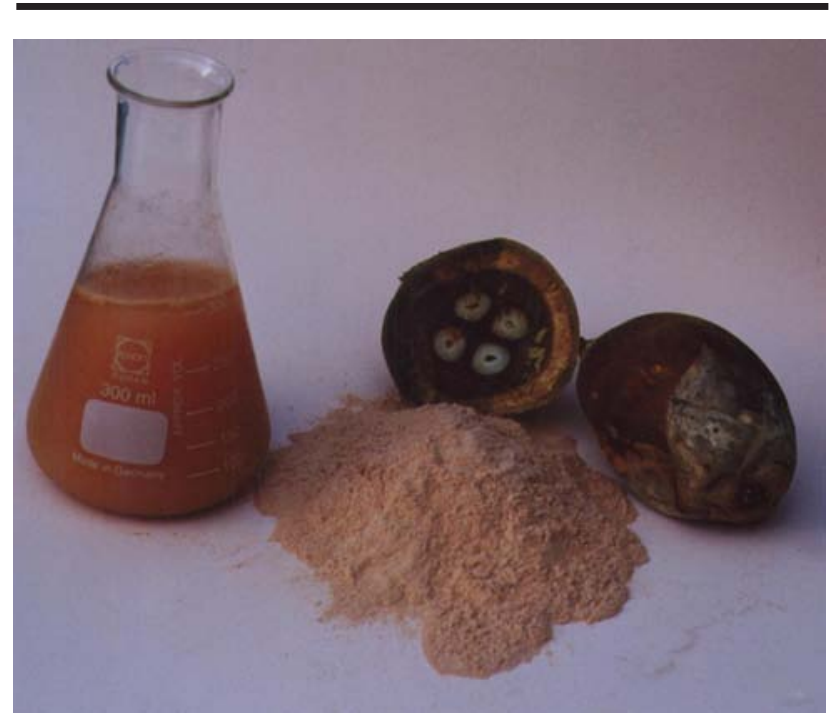

FIGURA 1 - Preparo do extrato do mesocarpo de Orbignya phalerata

utilizando o fio de polipropileno 6-0 em plano único, realizou-se a gastrorrafia.

Após este procedimento comum, os animais foram distribuídos aleatoriamente em dois grupos contendo 20 ratos cada, sendo o Grupo Controle (GC), que recebeu injeção intraperitoneal de água destilada na quantidade de $1 \mathrm{ml} / \mathrm{kg}$ de peso corpóreo e o Grupo Orbignya (GO), tratado com injeção intraperitoneal de extrato aquoso do mesocarpo de Orbignya phalerata, na dose de $50 \mathrm{mg} / \mathrm{kg}$ de peso corpóreo. A síntese da parede abdominal deu-se em dois planos com fio monofilamentar 5-0.

Os ratos foram, em seguida, identificados com ácido pícrico e cada grupo foi dividido em dois subgrupos contendo 10 animais cada, definidos como subgrupo controle três dias (SGC3), subgrupo controle 7 dias (SGC7), subgrupo Orbignya três dias (SGO3) e subgrupo Orbignya sete dias (SGO7), conforme a data da morte dos animais no terceiro e sétimo dias do período pós-operatório.

Os animais foram mantidos em laboratório recebendo ração padrão para ratos e água ad libitum e avaliados diariamente.

Nos dias determinados para aferição, os animais foram mortos e a ferida operatória examinada. Em seguida, a cavidade abdominal foi aberta e realizado o inventário. A peça foi retirada compreendendo $1 \mathrm{~cm}$ do esôfago distal, o estômago e $1 \mathrm{~cm}$ do duodeno proximal. As aderências encontradas eram retiradas em monobloco com a peça. Em seguida este segmento foi limpo com solução de cloreto de sódio a $0,9 \%$ e submetido ao teste de resistência à insuflação 
de ar atmosférico.

A avaliação do teste de resistência à insuflação de ar atmosférico consistiu em introdução de sonda de silicone $\mathrm{n}^{0}$ 6 no segmento esofágico e fixação desta com fio de algodão 2-0, conexão da sonda ao manômetro, submersão da peça em água, insuflação com ar ambiente a velocidade de $0,1 \mathrm{ml} / \mathrm{s}$ até a ocorrência de liberação de bolhas de ar, sendo registrado o momento da ruptura da peça em mmHg (Figura 2).

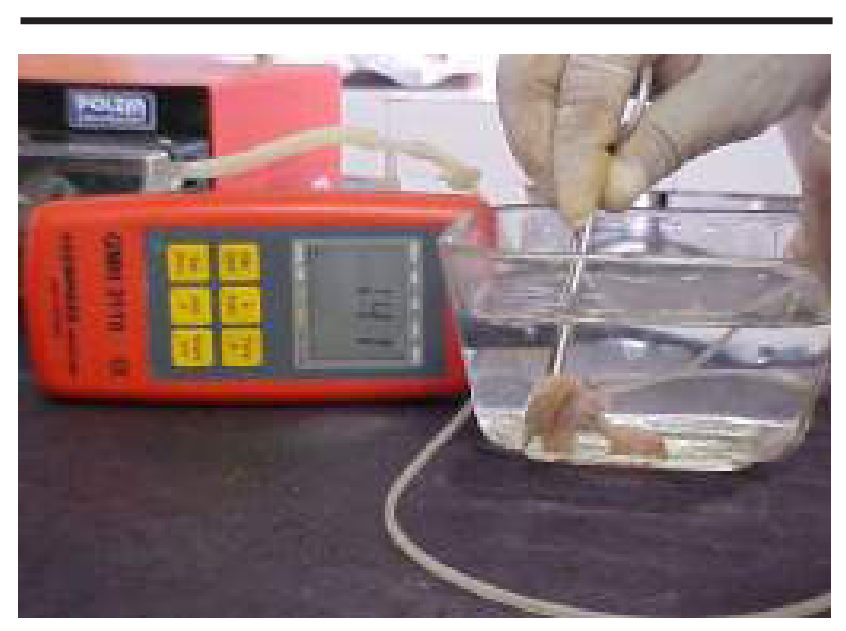

FIGURA 2 - Teste de insuflação de ar atmosférico

O segmento ressecado foi aberto na sua parede dorsal e seccionado em fragmentos, medindo 1,0 x 0,5 cm de área, mantendo sempre a anastomose no centro. A face interna da linha de sutura ficou voltada para cima, presa na placa de isopor, de $2 \mathrm{~cm}^{2}$, por meio de alfinetes. Fixado em formol a $10 \%$ por $48 \mathrm{~h}$ e encaminhado para estudo histológico, mantidas nos blocos de parafina e cortadas com micrótomo ajustados para espessura de $5 \mu \mathrm{m}$ e coradas pela Hematoxilina-Eosina (HE). As lâminas foram analisadas por um único médico patologista e por um dos autores deste trabalho (CPB) em microscópio óptico tetraocular, com ocular de 10x e objetivas de $4 \mathrm{x}$ e 10x.

Os dados foram analisados utilizando-se o programa Statistica for Windows 5.1. A pressão de insuflação de ar atmosférico foi analisada pelo teste t de Student. As variáveis das avaliações macroscópica e microscópica foram analisadas pelo teste não-paramétrico de Mann-Whitney. O nível de significância $(\alpha)$ utilizado para se rejeitar a hipótese da nulidade foi de $5 \%(\mathrm{p}<0,05)$.

\section{Resultados}

Todos os animais recuperaram-se bem da anestesia. Nas evoluções clínicas diárias, eles mantiveram-se ativos e com bom estado geral, não se observando óbito ou outra intercorrência.

A cicatrização da parede abdominal foi considerada boa em todos os animais, não sendo observados sinais clínicos de infecção, deiscência, fístula, seroma, hematoma ou evisceração. Na avaliação da cavidade abdominal também não foram notados abscessos, fístulas ou hematomas.

As aderências observadas estão representadas na Tabela 1.

Foi observada deiscência da gastrorrafia em um rato do SGO3, no momento da liberação da aderência desta com o fígado. Não foram observados abscessos ou fístulas nas peças cirúrgicas estudadas. $\mathrm{O}$ aspecto da mucosa no local da sutura foi considerado bom em todos os animais.O teste de resistência à insuflação de ar atmosférico não foi realizado no animal acima referido do SGO3 devido à deiscência da gastrorrafia. Nos demais, o vazamento de ar ocorreu na linha de sutura.

Os valores médios da pressão de resistência à insuflação de ar observados nos SGO3 e SGC3 foi de 26,8 mmHg e 41,2 $\mathrm{mmHg}$, respectivamente $(\mathrm{p}=0,087)$. A média da pressão de resistência à insuflação de ar no $\mathrm{SGO} 7$ foi de $105,3 \mathrm{mmHg}$ e no SGC7 foi de $117 \mathrm{mmHg}$ ( $\mathrm{p}=0,337)$.

A avaliação microscópica do $3^{\circ}$ dia do período pós-operatório não revelou diferença significativa quando comparados os SGO3 e SGC3. A coaptação das bordas esteve ausente em todas as gastrorrafias do SGC3 enquanto que nos animais do SGO3 havia coaptação completa em um animal e parcial em dois $(p=0,068)$.

A inflamação aguda no SGC7 mostrou-se moderada em oito animais e discreta em dois (Tabela 2, Figura 3). Enquanto que no $\mathrm{SGO} 7$, cinco animais apresentaram in-

TABELA 1 - Descrição da presença de aderências da superfície externa da gastrorrafia com a parede e órgãos intraperitoneais, por animal, de acordo com os subgrupos de estudo

\begin{tabular}{|c|c|c|c|c|}
\hline \multirow{2}{*}{ Animal } & \multicolumn{4}{|c|}{ Aderências (órgãos aderidos) } \\
\hline & SGO & $\mathrm{SGC}_{2}$ & $\mathrm{SGO}_{7}$ & $\mathrm{SGC}_{7}$ \\
\hline 1 & ------ & Fígado & Fígado & Fígado \\
\hline 2 & ------ & Fígado & ------ & ------ \\
\hline 3 & Fígado & Fígado/Parede abdominal & ------ & Fígado \\
\hline 4 & Fígado & Fígado & Fígado/Parede abdominal & Fígado \\
\hline 5 & Fígado & Fígado & Fígado/Parede abdominal & Fígado \\
\hline 6 & Parede abdominal & ------ & ------ & Fígado/Parede abdominal \\
\hline 7 & Parede abdominal & ------ & Fígado/Intestino delgado & Fígado \\
\hline 8 & Fígado/Parede abdominal & Fígado/Intestino delgado & Fígado/Parede abdominal & Fígado \\
\hline 9 & Fígado & Fígado & Fígado/Parede abdominal & Fígado \\
\hline 10 & Fígado & ----- & Fígado & Fígado \\
\hline $\mathbf{0} \pm \mathbf{Z}$ & $1 \pm 0,037$ & $1 \pm 0,037$ & $1,5 \pm 0,68$ & $1 \pm 0,68$ \\
\hline $\mathbf{U}$ & 49,5 & 49,5 & 41 & 41 \\
\hline
\end{tabular}


flamação discreta, quatro moderada e em um deles não houve inflamação aguda $(\mathrm{p}=0,066)$.

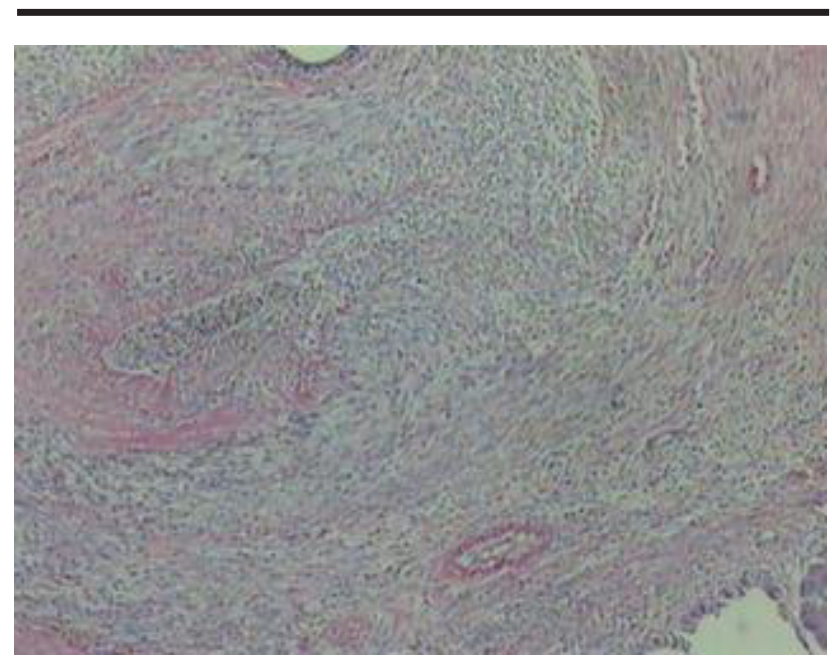

FIGURA 3 - Inflamação aguda moderada no SGC7

TABELA 2 - Intensidade da inflamação aguda nos animais dos subgrupos controle e Orbignya, analisada nos $3^{\circ} \mathrm{e}$ $7^{\circ}$ dias do período pós-operatório.

\begin{tabular}{ccccc}
\hline Inflamação & \multicolumn{4}{c}{ Subgrupo } \\
\cline { 2 - 5 } aguda & $\mathbf{S G O}_{2}$ & $\mathbf{S G C}_{\mathbf{2}}$ & $\mathbf{S G O}_{\mathbf{7}}$ & $\mathbf{S G C}_{7}$ \\
\hline $\mathbf{1}$ & & & & \\
$\mathbf{2}$ & & & 1 & 2 \\
$\mathbf{3}$ & 6 & 8 & 5 & 8 \\
$\mathbf{4}$ & 4 & 2 & 4 & \\
Total & 10 & 10 & 10 & 10 \\
\hline $\mathbf{0} \pm \mathbf{Z}$ & $\mathrm{c}$ & $1 \pm 0,95$ & $2 \pm 1,84$ & $2 \pm 1,84$ \\
$\mathbf{U}$ & 40 & 40 & 29 & 29 \\
\hline
\end{tabular}

A inflamação crônica foi classificada como grau 1 nos animais dos dois grupos no $3^{\circ}$ dia, e como grau 3 em dois animais do SGC7 (Tabela 3).

TABELA 3 - Intensidade da inflamação crônica nos animais dos subgrupos controle e Orbignya, analisada nos $3^{\circ} \mathrm{e}$ $7^{\circ}$ dias do período pós-operatório.

\begin{tabular}{ccccc}
\hline Inflamação & \multicolumn{4}{c}{ Subgrupo } \\
\cline { 2 - 5 } crônica & $\mathbf{S G O}_{\mathbf{3}}$ & $\mathbf{S G C}_{\mathbf{3}}$ & $\mathbf{S G O}_{\mathbf{7}}$ & $\mathbf{S G C}_{\mathbf{7}}$ \\
\hline $\mathbf{1}$ & 8 & 8 & & \\
$\mathbf{2}$ & 2 & 2 & 10 & 8 \\
$\mathbf{3}$ & & & & 2 \\
$\mathbf{4}$ & & & & \\
Total & 10 & 10 & 10 & 10 \\
\hline $\mathbf{0} \pm \mathbf{Z}$ & $3 \pm 0,00$ & $3 \pm 0,00$ & $2 \pm 1,45$ & $3 \pm 1,45$ \\
$\mathbf{U}$ & 50 & 50 & 40 & 40 \\
\hline
\end{tabular}

A neoformação capilar mostrou-se discreta em nove animais e moderada em um animal do SGO7 (Figura 4). No SGC7, ela apresentou-se discreta em cinco animais, moderada em quatro e acentuada em um $(p=0,053)$ (Tabela 4).

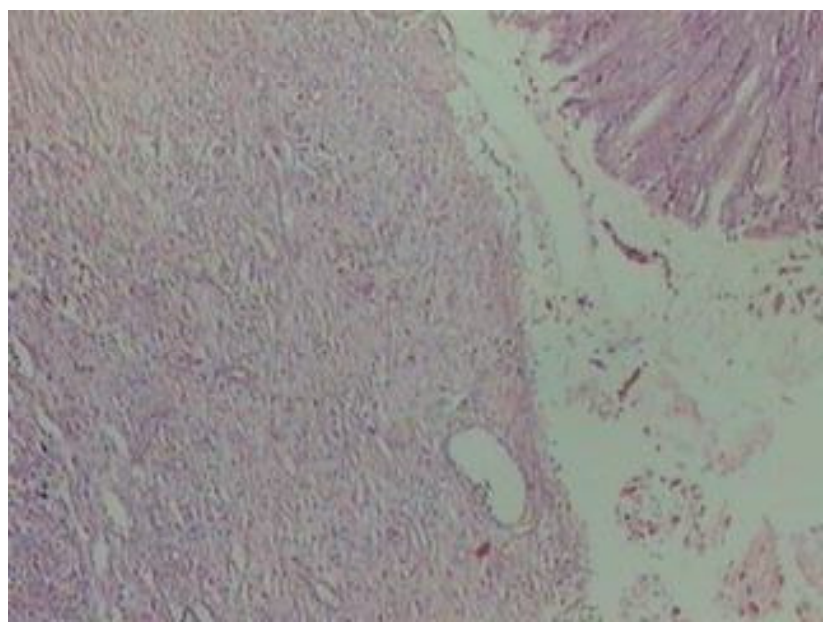

FIGURA 4 - Neoformação capilar discreta SGO7

TABELA 4 - Intensidade da neoformação capilar nos animais dos subgrupos controle e Orbignya, analisada nos $3^{\circ}$ e $7^{\circ}$ dias do período pós-operatório

\begin{tabular}{ccccc}
\hline \multirow{2}{*}{$\begin{array}{c}\text { Neoformação } \\
\text { capilar }\end{array}$} & $\mathbf{S G O}_{3}$ & $\mathbf{S G C}_{2}$ & $\mathbf{S G O}_{7}$ & $\mathbf{S G C}_{\mathbf{7}}$ \\
\cline { 2 - 5 } & 10 & 8 & & \\
$\mathbf{1}$ & & 2 & 9 & 5 \\
$\mathbf{3}$ & & & 1 & 4 \\
$\mathbf{4}$ & & & & 1 \\
Total & 10 & 10 & 10 & 10 \\
\hline $\mathbf{0} \pm \mathbf{Z}$ & $1 \pm 1,45$ & $1 \pm 1,45$ & $2 \pm 1,93$ & $2,5 \pm 1,93$ \\
$\mathbf{U}$ & 40 & 40 & 29 & 29 \\
\hline
\end{tabular}

No SGO7 a coaptação das bordas foi completa em todos os animais (Figura 5). No SGC7, completa em um animal, parcial em oito e em um não houve coaptação $(p=0,000$, Tabela 5).

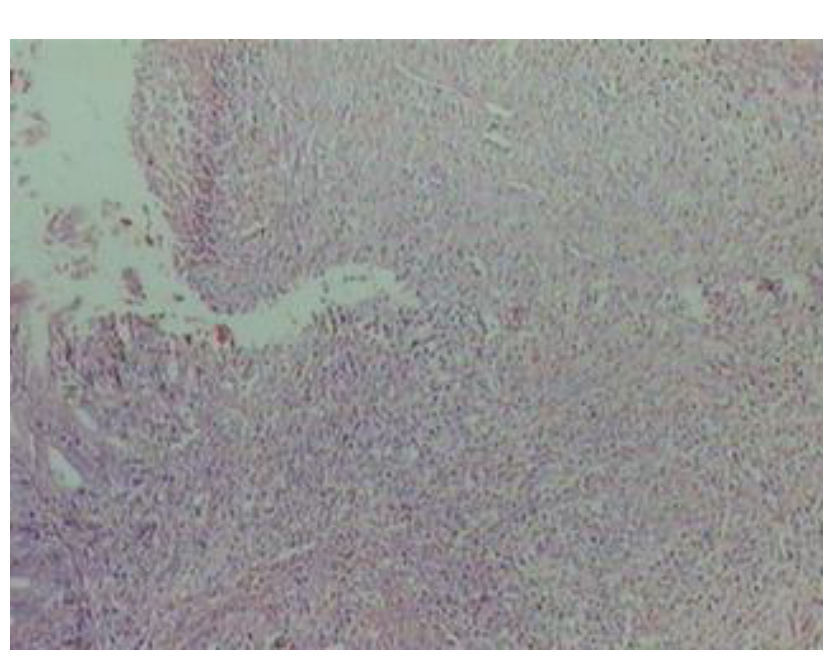

FIGURA 5 - Neoformação capilar discreta SGO7 
TABELA 5 - Intensidade da coaptação das bordas nos animais dos subgrupos controle e Orbignya, analisada nos $3^{\circ}$ e $7^{\circ}$ dias do período pós-operatório

\begin{tabular}{ccccc}
\hline $\begin{array}{c}\text { Coaptação } \\
\text { das bordas }\end{array}$ & $\mathbf{S G O}_{\mathbf{3}}$ & $\mathbf{S G C}_{\mathbf{3}}$ & $\mathbf{S G O}_{7}$ & $\mathbf{S G C}_{\mathbf{7}}$ \\
\cline { 2 - 5 } $\mathbf{1}$ & 7 & 10 & & 1 \\
$\mathbf{2}$ & 2 & & & 8 \\
$\mathbf{3}$ & 1 & & 10 & 1 \\
$\mathbf{4}$ & & & & \\
Total & 10 & 10 & 10 & 10 \\
\hline $\mathbf{0} \pm \mathbf{Z}$ & $1 \pm 1,83$ & $1 \pm 1,83$ & $3 \pm 3,87$ & $2 \pm 3,87$ \\
$\mathbf{U}$ & 35 & 35 & 5 & 5 \\
\hline
\end{tabular}

\section{Discussão}

O extrato clorofórmico do mesocarpo de Orbignya phalerata, na dose de $50 \mathrm{mg} / \mathrm{kg}$ administrado por via intra-peritoneal apresentou atividades analgésica e antiinflamatória comparáveis a da fenilbutazona na dose de $100 \mathrm{mg} / \mathrm{kg}$ por via oral. Não foram observados efeitos sedativo, narcótico ou antipirético, bem como lesões gástricas, em vários modelos experimentais ${ }^{1,4,5,8,9,10}$.

Alguns estudos observaram que o extrato hidroalcoólico do mesocarpo de Orbignya phalerata foi capaz de estimular a produção de anticorpos auto-reativos em camundongos. Outros, utilizando camundongos, constataram que o extrato aquoso do mesocarpo de Orbignya phalerata induziu a significativo aumento do número de células tumorais no peritônio e do número de linfócitos respondedores à bromelina, não sendo observadas, entretanto, atividades analgésica ou antiinflamatória ${ }^{1,3,4,5,8}$.

O extrato aquoso do mesocarpo de Orbignya phalerata apresenta, em sua composição, substâncias capazes de estimular a produção de anticorpos auto-reativos e pode representar um fator desencadeante e/ou agravante das doenças auto-imunes como diabetes e tireopatias ${ }^{1,5,8,11,13}$.

O uso popular do mesocarpo de Orbignya phalerata como alimento e no tratamento de diversas enfermidades, bem como a sua ampla distribuição pelo território maranhense motivaram a escolha do extrato do mesocarpo como substância a ser avaliada neste estudo. Não se observaram, na literatura pesquisada, estudos que avaliassem a ação cicatrizante do mesocarpo de Orbignya phalerata, entretanto, outros estudos têm demonstrado atividades antiinflamatória, imunomoduladora, analgésica e antipirética ${ }^{3,4,6,11}$. Esses autores também constataram facilidade de obtenção, preparo, estocagem e utilização do extrato de Orbignya phalerata.

Pela facilidade de acesso, escolheu-se a parede anterior do estômago do rato para a realização da incisão cirúrgica. Por ser um órgão oco, pode-se avaliar a resistência da cicatrização nas fases inicial e intermediária, através do teste de ruptura por insuflação de ar atmosférico. Empregou-se a sutura em plano único e com pontos separados por ser técnica de anastomose prática e segura ${ }^{6,7,12,14,15,16,17}$.

Utilizou-se para a sutura da incisão gástrica o fio de polipropileno que apresenta facilidade de fixação de nós e pouca reação inflamatória, levando à formação de pouco tecido de granulação, mesmo nas vísceras ocas, sujeitos a aumento de pressão intraluminal, não interferindo conse- quentemente na proposta do estudo. Segundo Nigro et al. ${ }^{6}$, o fio de polipropileno é o material de síntese mais adequado para as gastrorrafias.

A síntese abdominal foi realizada através de sutura contínua por apresentar bons resultados em modelos experimentais. A dieta hídrica e alimentar foi liberada no pós-operatório imediato, de acordo com a recuperação anestésica. A disposição para se alimentar e a manutenção do bom estado geral dos animais sugeriram boa evolução no processo cicatricial das suturas gástricas. Fato este corroborado pelas avaliações macroscópicas e microscópicas das peças cirúrgicas.

O terceiro dia do período pós-operatório foi escolhido para avaliação da cicatrização gástrica por representar fase inicial e crítica da reparação tecidual, esperando-se encontrar processo inflamatório intenso e baixa resistência à insuflação de $\operatorname{ar}^{7,12,17}$. Em torno do sétimo dia do período pós-operatório ocorrem as principais complicações das anastomoses gástricas, quais sejam: deiscência, extravasamento e fístulas êntero-cutâneas, e por isso optou-se por realizar avaliação nesta data.

Os achados do aspecto da ferida operatória e da cavidade peritoneal em todos os animais estudados não diferiram. A análise dos parâmetros da cicatrização deve ser feita levando-se em consideração a determinação da resistência mecânica da cicatriz, o estudo histológico da morfologia tecidual e a determinação da taxa de colágeno ${ }^{2,7,12,18}$.

Neste estudo utilizou-se o teste de resistência à insuflação de ar atmosférico para avaliar a resistência mecânica da cicatriz. Quando se pretende avaliar fisicamente uma cicatriz tecidual, deve-se levar em consideração o tipo de força natural a que este tecido está sujeito. Sendo o objetivo do presente estudo o trato digestório, o teste de resistência à insuflação de ar atmosférico constitui-se em parâmetro fisiológico e imprescindível para esta análise, por produzir os vetores de pressão que normalmente se transmitem sobre a parede do intestino, levando à ruptura das anastomoses das vísceras ocas por distensão $0^{2,14,15,16,17}$.

$\mathrm{O}$ ar insuflado na víscera oca exerce pressão circunferencial na parede intestinal, dando idéia de vedação da anastomose. Este teste deve ser realizado nos primeiros dias do período pós-operatório, pois a partir da segunda semana, a resistência da anastomose à pressão pode exceder a do tecido intestinal normal e o escape gasoso ocorrer distante da anastomose $\mathrm{e}^{7,12}$

Neste estudo procurou-se não se desfazer as aderências da peça cirúrgica para não prejudicar os resultados, pela manipulação indevida da anastomose. Este procedimento é sugerido por Hendriks ${ }^{14}$. Entretanto, em um dos animais ocorreu deiscência parcial da gastrorrafia no momento da retirada da peça cirúrgica, inviabilizando a aplicação deste teste.

Observou-se nesta pesquisa resistência à insuflação de ar atmosférico mais baixa no $\mathrm{SGO} 3$ quando comparado ao SGC3 (médias de 26,8 $\mathrm{mmHg}$ e 41,2 $\mathrm{mmHg}$, respectivamente) mostrando diferença marginalmente significativa $(\mathrm{p}=0,087)$. A análise das médias de pressão no sétimo dia do período pós-operatório demonstrou pressão maior do que a observada no $3^{\circ}$ dia $(\mathrm{SGO} 7=105,3 \mathrm{mmHg}$ e SGC7 $=117$ 
$\mathrm{mmHg}$ ), resultados esperados pela baixa resistência devido à colagenólise maior neste período, porém sem diferenças significativas $(\mathrm{p}=0,337)$, quando comparados os subgrupos Orbignya e controle ${ }^{19,20}$.

No presente trabalho, avaliaram-se comparativamente vários parâmetros histológicos da reparação tecidual, quais sejam: inflamação aguda, inflamação crônica, necrose isquêmica, reação gigantocelular do tipo corpo estranho, neoformação capilar, proliferação fibroblástica, fibrose, reepitelização, coaptação das bordas e extensão da inflamação na parede.

Ambos os grupos, no $3^{\circ}$ dia do período pós-operatório, apresentaram inflamação aguda de moderada a acentuada. A inflamação aguda em certo grau, é necessária para boa resposta de reparação tecidual, entretanto, reação inflamatória intensa pode diminuir o suprimento sangüíneo, com conseqüente inibição da proliferação fibroblástica. A coaptação das bordas foi observada em três animais do SGO3 $(p=0,068)$, sugerindo diferença marginalmente significativa, favorecendo este subgrupo.

No $7^{\circ}$ dia do período pós-operatório houve diferença estatisticamente significativa na variável coaptação das bordas $(p=0,000)$, que foi completa em todos os animais do SGO7. Diferença marginalmente significativa foi notada na inflamação aguda $(p=0,066)$ e na neoformação capilar $(p=0,053)$, sugerindo que um tempo de observação maior pudesse apontar vantagens do uso de Orbignya phalerata, em relação a esses parâmetros.

\section{Conclusões}

Observou-se que o extrato aquoso do mesocarpo de $\mathrm{Or}$ bignya phalerata, na dose de $50 \mathrm{mg} / \mathrm{kg}$, por via intraperitoneal em ratos foi capaz de favorecer completa coaptação de bordas da cicatriz gástrica, quando comparada ao grupo controle, nos animais mortos no $7^{\circ}$ dia do período pós-operatório. Nos demais parâmetros analisados não foi possível observar diferenças estatisticamente significantes entre os grupos de estudo.

\section{Referências}

1. Barroqueiro ESB. Efeito do mesocarpo de babaçu sobre a produção de anticorpos auto-reativos. [Dissertação- Mestrado]. São Luis: Universidade Federal do Maranhão, Centro de Ciências Biológicas e da Saúde; 2001.

2. Brito MVH, Oliveira RVB, Morais MR, Miranda JS, Reis JMC. Análise gástrica após administração de copaíba: estudo experimental em ratos. GED. 2001; 20: 78-82.

3. Chagas AP. Atividade do mesocarpo babaçu (Orbignya phalerata) sobre o desenvolvimento tumoral e a população de linfócitos B. [Monografia-Graduação]. São Luis: Universidade Federal do Maranhão, Centro de Ciências Biológicas e da Saúde; 2001.

4. Gaitan E, Cooksey RC, Legan J, Lindsay RH, Ingbar $\mathrm{SH}$, Medeiros-Neto G. Antithyroid effects in vivo and in vitro of babassu and mandioca: a staple food in goiter areas of Brazil. Eur J Endocrinol. 1994; 131:138-44.

5. Guerra RNM, Brito RM, Maia MS, Soares SM. Aumento da produção de auto-anticorpos em animais tratados com extrato bruto de babaçu ou com sua fração metanólica. In: XIII Reunião Anual da Federação de Sociedades de Biologia Experimental - FESBE, 1998, Caxambu. Reunião Anual da Federação de Sociedades de Biologia Experimental - FeSBE. São Paulo: FESBE, 1998;1:390.

6. Nigro AJT, Hirota RT, Paula RM, Simões AGS, Vieira WTT. Estudo comparativo em cães das gastrorrafias feitas com fios de polipropileno ou de ácido poliglicólico. Acta Cir Bras. 1993;8:118-23.

7. Thornton FJ, Barbul A. Healing in the gastrointestinal tract. Surg Clin Cir Am North. 1997; 547-70.

8. Brito RMF. Avaliação da auto-reatividade induzida pelo tratamento com babaçu. [Monografia-Graduação]. São Luís: Universidade Federal do Maranhão, Centro de Ciências Biológicas e da Saúde; 2001.

9. Oliveira AB. Estudo fitoquímico do mesocarpo do coco de babaçu (Orbignya phalerata Mart). [MonografiaEspecialização]. São Luis: Universidade Federal do Maranhão. Centro tecnológico;1998.

10. Rosenthal FRT. O amido do côco babaçu, algumas propriedades dos grânulos e das pastas. Rev Bras Tecnol. 1975; 6:6-29.

11. Silva BP, Parente P. An anti-inflammatory and immunomodulatory polisaccharide from Orbignya phalerata. Fitoterapia. 2001;72:887-93.

12. Singer AJ, Clark RAF. Cutaneous wound healing. New England J Med. 1999; 341:746-8.

13. Maia MBS. Estudo de atividade anti-inflamatorio e outros efeitos farmacológicos relacionados de orbignya phalerata Mart. [Dissertação-Mestrado]. Fortaleza: Universidade Federal do Ceará, Departamento de Fisiologia; 1987.

14. Hendriks T, Mastboom WJB. Healing of experimental intestinal anastomoses. Parameters for repair. Dis Colon Rectum. 1990;33:891-901.

15. Kodura MJ, Rolandelli RH. Current research review: experimental studies on the healing of colonic anatomoses. J Surg Res. 1990;48:504-15.

16. Moraes SP, Melro APC, Longo A, Gun PR, Silva MAJ, Neto JARN. Influência de betabloqueadores H2 no processo de cicatrização gástrica. Rev Col Bras Cir.1999;26: 153-5.

17. Petroinau A, Cavalcante AMT, Fagundes-Pereyra WJ, Santos BMR. Tensão de ruptura dos órgãos que constituem o tubo digestivo com e sem o uso de corticóide em camundongos. Rev Col Bras Cir. 1999;27:69-72.

18. Ramzi S. Robins basis of disease. Philadelphia: W.B. Saunders Company, 6ed, 1999.

19. Warde PJ. Anastomose colorretal experimental em um e em dois planos de sutura: estudo comparativo. São Paulo, 1972. [Tese- Doutorado]. São Paulo: Universidade de São Paulo: Faculdade de Medicina; 1972.

20. Maia MBS. Estudo da atividade antiinflamatória e outros efeitos farmacológicos relacionados de Orbignya phalerata Mart [Dissertação-Mestrado]. Fortaleza: Universidade Federal do Ceará; 1987. 
Recebimento: $21 / 01 / 2005$

Revisão: 24/05/2005

Aprovação: 22/01/2006

\section{Como citar este artigo:}

Batista CP, Torres OJM, Matias JEF, Moreira ATR, Colman D, Lima JHF, Macri MM, Rauen Jr RJ, Ferreira LM, Fraitas ACT. Efeito do extrato aquoso de Orbignya phalerata (babaçu) na cicatrização do estômago em ratos: estudo morfológico e tensiométrico. Acta Cir Bras. [periódico na internet] 2006;21 Supl 3:26-32. Disponível em URL: http://www.scielo. $\underline{\mathrm{br} / \mathrm{acb}}$

*Figuras coloridas disponíveis em www.scielo.br/acb 